\title{
V6 - Characterization of native and deacylated Lipid A from lipooligosaccharide of Neisseria meningitidis Serogroup B
}

Marilza B. Corrêa ${ }^{1 \star}$; Maria de Lourdes M. Leal ${ }^{1}$; Elza F. Scott ${ }^{1}$; Adenilza Amancio Bello ${ }^{1}$; Eduardo Duarte ${ }^{1}$; Ellen Jessouroun ${ }^{1}$.

\section{1 - Bio-Manguinhos/FIOCRUZ}

\section{Introduction:}

The Brazilian vaccine against meningococcal disease Neisseria meningitidis serogroup B is composed by outer membrane vesicles (OMV) and chemically modified endotoxin. Which the last aims to improve the efficiency of the vaccine. Lipooligosaccharide (LOS) is one of the major outer membrane constituents of all Gram-negative bacteria. It is formed by an oligosaccharide core covalently linked to Lipid A, which consists of a diglucosamine backbone carrying two diphosphorylethanolamine in 1 and 4' positions and hexa-acyl substituents. There is a straight relationship between Lipid A structure and its inflammatory activity. It's known that the removal of acyl chains and the hydrolysis of diphosphoryl-ethanolamine esters reduce drastically its inflammatory activity. Due to its high toxicity a safe protocol of kinetic detoxification is required, which involves a pyrogenic monitoring and LOS characterization.

\section{Objective:}

The aim of this study is to characterize native and deacylated Lipid A, from Neisseria meningitidis serogroup B using different analytical approach as mass spectrometry (ESI-MS ${ }^{\mathrm{n}}$ and MALDI), NMR HMQC ${ }^{1} \mathrm{H} \times{ }^{31} \mathrm{P}$, CGAR and CG-MS.

\section{Methodology:}

The LOS was obtained from bacteria biomass, purified by hot phenol method. The native LOS was hydrolyzed in acetate buffer and native Lipid A was recovered by ultracentrifugation and its molecular weight was determinated by MALDI/TOF and ESI-MS ${ }^{n}$, operating in positive mode. To characterize the acyl chains, an alkaline hydrolysis was performed, the fatty acid esterified and analyzed by GC-MS. The kinetic detoxification of LOS in alkaline media was performed in different times and monitored by pyrogenic test and the structures analyzed by CE-MS and NMR HSQC ${ }^{1} \mathrm{H} \mathrm{x}^{31} \mathrm{P}$. 


\section{Results:}

Mass spectroscopy was used as an effective technique for analyzing lipid A and dLOS. The molar weight of the major lipid was characterized by MALDI-TOF; the analysis of ion $[\mathrm{M}+\mathrm{H}]+$ at $\mathrm{m} / \mathrm{z}$ 1960,98 that was correlated to a hexa-acylated 1,4'-diphosphoryl-ethanolamine and this result is in accordance to ESI $\mathrm{MS}^{\mathrm{n}}$ analysis. The acyl chains were analyzed as fatty acid methyl esters by GC -MS (IE, 70ev), and the results were in accordance with Neisseriaceae family. The kinetic detoxification of LOS was carried out with $\mathrm{NaOH}$ at 30,60, 90, 120 and 150 minutes. the dLOS molecular weight was analyzed by CE-MS and the majority deacylated lipid A comprised a diphosphorylated molecule with two amide-linked 3-hydroxytetradecanoic acids and phosphate groups in 1,4'. The HMQC ${ }^{1} \mathrm{H} \mathrm{x}^{31} \mathrm{P}$ showed cross resonance consistent with 5.6/-12.0; 5.4/2.0 and 3.7/4.9 ppm, indicating that there are two different phosphorous species in anomeric position and diphosphate in $4^{\prime}$.

\section{Conclusion:}

The results allowed us to identify the phosphorylation and acylation pattern of lipid A. These data are important for the use of dLOS as vaccinal antigen.

Keywords: LOS, Neisseria meningitidis, Lipid A 